\section{FAST, QUIET AMALGAMATOR}

The Ultramat 2 amalgamator's robust and reliable construction comes with a rock solid 5-year warranty.

The Ultramat 2 is the quietest low vibration mixer available on the market today. It features a 0-16 second microprocessor-crystal controlled oscillator timer, which provides consistent and accurate trituration, ensuring the optimal handling and clinical characteristics of mixed materials. The high energy amalgamator has a mixing frequency of 4,600 oscillations per minute $+/-1 \%$ even though it has economical low energy usage.

The touch control system of the Ultramat 2 is quick and simple to operate. The one surface cover facilitates infection control cleaning and the nonporous plastic surface can be cleaned with any dental disinfectant.

The safety micro-switch automatically halts trituration when the lid of the Ultramat 2 is lifted.

The multi-use Ultramat 2 is suitable for all types of encapsulated dental materials, particularly amalgam and glass ionomer capsules.

\section{Reader response number 57}

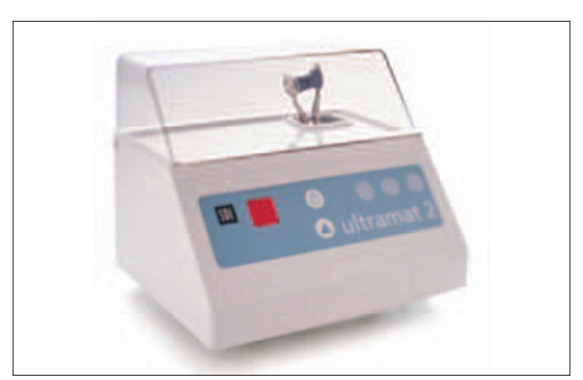

\section{POWERFUL PROGRAMS}

Carestream Dental's R4 practice management software offers dental practices a powerful suite of programs designed to make workflow more efficient and help improve the overall patient experience.

Included free with every $\mathrm{R} 4$ operating software bundle is R4 Communicator, a sophisticated yet user-friendly dental communication package that makes light work of patient education sessions.

Employing one of the 200 high quality computer animation sequences, as well as the built-in sketch function, dental professionals can quickly and easily explain a number of procedures and conditions to patients, increasing treatment acceptance rates and helping to overcome communication barriers.

In addition, R4 Communicator also includes a range of over 120 related patient information leaflets, 40 of which were compiled by the British Dental Health Foundation. Together with the animations, these can be emailed to or printed out for the patient, ensuring the patient experience continues even after they have left the surgery. Because Communicator is fully integrated into R4 practice management software, all the information provided to patients is automatically recorded into their patient notes, making consent protocols even easier to document.

Communicator covers preventative, restorative and oral hygiene topics, with a special tool available for orthodontists. Thanks to regular improvements, including the introduction of a new section of implant sequences, Communicator has something to offer every type of clinician.

Reader response number 60 wrap-around titanium sport frame. ExamVision loupes are manufactured using superior materials and optics to provide optimal magnification, maximum sharpness and true colour transmission. ExamVision loupes and lights are available from Evident, the UK's No.1 supplier of loupes and lights. Reader response number 59

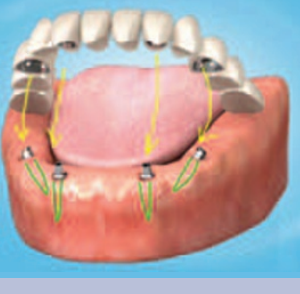

